\title{
The Stability of Solitary Waves of Depression
}

\author{
Nguyen Thanh Nguyet and Henrik Kalisch
}

\begin{abstract}
We provide a sufficient condition for the orbital stability of negative solitary-wave solutions of the regularized long-wave equation. In particular, it is found that solitary waves with speed $c<-\frac{1}{6}$ are orbitally stable.
\end{abstract}

Mathematics Subject Classification (2000). Primary 35Q53; Secondary 35B35.

Keywords. Solitary waves, orbital stability, long wave equations.

\section{Introduction}

In this article, the dynamic stability of negative solitary-wave solutions of the regularized long-wave equation

$$
u_{t}+u_{x}+\left(u^{2}\right)_{x}-u_{x x t}=0,
$$

is investigated. This equation which is also called the BBM equation, is used to model the propagation of small-amplitude surface waves on a fluid running in a long narrow channel. For an account of modeling properties of (1.1), the reader may consult the work of Benjamin et al. [6], Peregrine [14] and Whitham [17]. As is well known, equation (1.1) admits solitary-wave solutions of the form $u(x, t)=\Phi(x-c t)$. Indeed, when this ansatz is substituted into (1.1), there appears the ordinary differential equation

$$
-c \Phi+\Phi+c \Phi^{\prime \prime}+\Phi^{2}=0
$$

where $\Phi^{\prime}=\frac{d \Phi}{d \xi}$, for $\xi=x-c t$. It is elementary to check that a solution of (1.2) is given by

$$
\Phi(\xi)=\frac{3}{2}(c-1) \operatorname{sech}^{2}\left(\frac{1}{2} \sqrt{\frac{c-1}{c}} \xi\right) .
$$

These solutions are strictly positive progressive waves which propagate to the right without changing their profile over time. As can be seen from the expression (1.3), solitary waves with positive propagation velocity are defined only when $c>1$. It is

This work was supported in part by the Research Council of Norway and the ESF Research Networking Programme HCAA. 
well known that these positive solitary waves are dynamically stable with respect to small perturbations. As observed by one of the authors in [12], the formula (1.3) is still valid when $c<0$, resulting in a solitary wave of depression which propagates to the left. Surprisingly, the stability of these solitary-waves of depression depends on the speed $c$. In fact, it was shown in [12] that for negative values of $c$ close to zero, the solitary waves are unstable.

The original proof of stability of positive solitary waves was given by Benjamin [5] and Bona [7], using previous ideas of Boussinesq concerning the characterization of solitary waves as extremals of a constrained minimization problem $[1,9]$. While the main thrust of their work was in the direction of the KortewegdeVries equation

$$
u_{t}+u_{x}+\left(u^{2}\right)_{x}+u_{x x x}=0
$$

their proof is also applicable to the regularized long-wave equation (1.1). In fact, the proof of stability of positive solitary waves appears in the appendix of [5]. The method of Benjamin has subsequently been refined and extended, and a general theory has been developed $[2,4,8,10,11,16]$. It appears however that almost all previous work has exclusively focused on positive solitary waves. In order to treat negative solitary waves, the general theory developed in $[10,16]$ cannot be applied straightforwardly, and it is our purpose here to indicate a complete proof of stability of negative solitary waves. Thus the main contribution of the present article is the proof of the following theorem.

Theorem. The solitary wave $\Phi$ with velocity $c$ is stable if $c<-\frac{1}{6}$.

Observe that this theorem provides a sufficient condition for the stability of negative solitary waves. We must hasten to mention however that numerical computations in $[12,13]$ suggest that our result is not sharp. For the sake of clarity, we closely follow the original proof of Benjamin without paying much heed to the more general theory.

Figure 1 is depicting a stable solitary wave of depression, with velocity $c=$ -1.2 and amplitude $\max _{x}|\Phi|=3.3$, propagating to the left.

\section{Preliminaries}

As already observed by Benjamin and others [5, 6], a solitary wave cannot be stable in the strictest sense of the word. To understand this, consider two solitary-waves of different height, centered initially at the same point. Since the two waves have different amplitudes, they have different velocities according to the formula (1.3). As time passes the two waves will drift apart, no matter how small the initial difference was. However, in the situation just described, it is evident that two solitary waves with slightly differing height will stay similar in shape during the time evolution. Measuring the difference in shape therefore will give an acceptable notion of stability. This sense of orbital stability was introduced by Benjamin [5]. We say the solitary wave is orbital stable, if for a solution $u$ of the equation (1.1) 


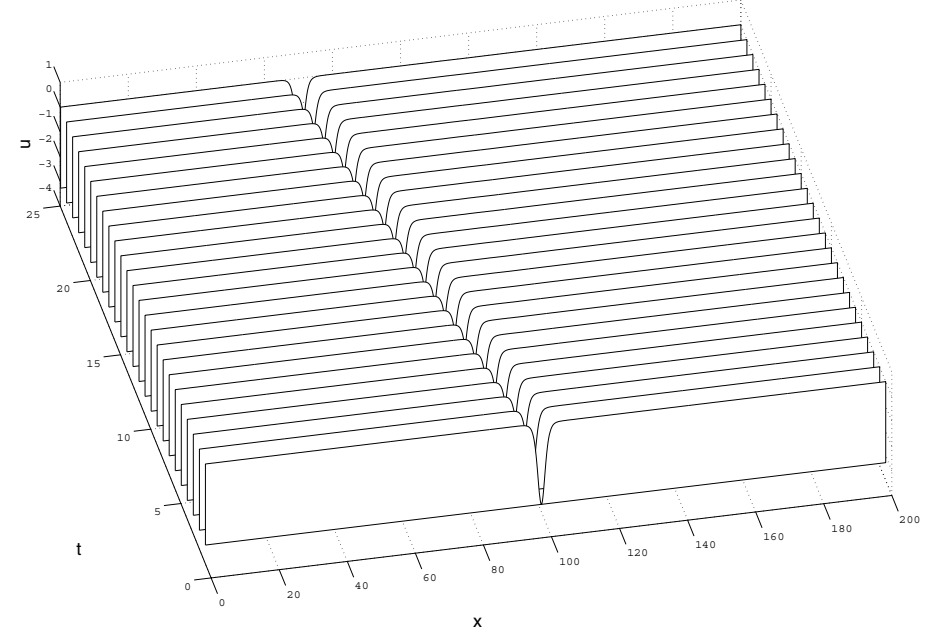

FIGURE 1. Stable solitary wave of depression with velocity $c=-1.2$.

that is initially sufficiently close to a solitary-wave will always stay close to a translation of the solitary-wave during the time evolution. A more mathematically precise definition is as follows. For any $\epsilon>0$, consider the tube

$$
U_{\epsilon}=\left\{u \in H^{1}: \inf _{s}\left\|u-\tau_{s} \Phi\right\|_{H^{1}}<\epsilon\right\},
$$

where $\tau_{s} \Phi(x)=\Phi(x-s)$ is a translation of $\Phi$. The set $U_{\epsilon}$ is an $\epsilon$-neighborhood of the collection of all translates of $\Phi$.

Definition 2.1. The solitary wave is stable if for any $\epsilon>0$, there exists $\delta>0$ such that if $u_{0}=u(\cdot, 0) \in U_{\delta}$, then $u(\cdot, t) \in U_{\epsilon}, \forall t \in \mathbb{R}$. The solitary wave $\Phi$ is unstable if $\Phi$ is not stable.

The proof of stability is based on the conservation of certain integral quantities under the action of the evolution equation. Equation (1.1) has four invariant integrals. In particular, the functionals

$$
V(u)=\frac{1}{2} \int_{-\infty}^{\infty}\left(u^{2}+u_{x}^{2}\right) d x
$$

and

$$
E(u)=\int_{-\infty}^{\infty}\left(\frac{1}{2} u^{2}+\frac{1}{3} u^{3}\right) d x
$$

are critically important to the proof of stability of $\Phi$. Note that $V(g)=\frac{1}{2}\|g\|_{H^{1}}^{2}$. The properties of these functionals are summarized in the following proposition. 
Proposition 2.2. Suppose $u$ is a smooth solution of (1.1) with sufficient spatial decay. Then $V$ and $E$ are constant as functions of $t$, invariant with respect to spatial translations and continuous with respect to the $H^{1}(\mathbb{R})$-norm.

Proof. The proof is standard. To see conservation in time for $V$ and $E$, multiply the equation by $u$ and $\left(-u-u^{2}+u_{x t}\right)$, respectively. Translation invariance means

$$
V(u)=V\left(\tau_{s}(u)\right) ; \text { and } E(u)=E\left(\tau_{s}(u)\right), \forall s \in \mathbb{R} .
$$

This follows immediately from the definition. Finally, we prove continuity of $E$ with respect to the $H^{1}(\mathbb{R})$-norm. Let $\left\{w_{n}\right\}$ be any sequence in $H^{1}(\mathbb{R})$ such that $\lim _{n \rightarrow \infty}\left\|w_{n}-w\right\|_{H^{1}}=0$. Then

$$
\begin{aligned}
& \left|E\left(w_{n}\right)-E(w)\right|=\left|\frac{1}{2} \int_{-\infty}^{\infty}\left[w_{n}^{2}-w^{2}\right] d x+\frac{1}{3} \int_{-\infty}^{\infty}\left[w_{n}^{3}-w^{3}\right] d x\right| \\
& \quad \leq \frac{1}{2}\left|\int_{-\infty}^{\infty}\left(w_{n}-w\right)\left(w_{n}+w\right) d x\right|+\frac{1}{3}\left|\int_{-\infty}^{\infty}\left(w_{n}-w\right)\left(w_{n}^{2}+w_{n} w+w^{2}\right) d x\right| .
\end{aligned}
$$

Using the Cauchy-Schwarz inequality, this can be dominated by

$$
\frac{1}{2}\left\|w_{n}-w\right\|_{L^{2}}\left\|w_{n}+w\right\|_{L^{2}}+\frac{1}{3}\left\|w_{n}-w\right\|_{L^{2}}\left\|w_{n}^{2}+w_{n} w+w^{2}\right\|_{L^{2}} .
$$

Thus there appears the estimate

$$
\begin{aligned}
& \left|E\left(w_{n}\right)-E(w)\right| \\
& \leq\left\|w_{n}-w\right\|_{H^{1}}\left\{\frac{1}{2}\left(\left\|w_{n}\right\|_{H^{1}}+\|w\|_{H^{1}}\right)+\frac{1}{3}\left(\left\|w_{n}^{2}\right\|_{H^{1}}+\left\|w_{n} w\right\|_{H^{1}}+\left\|w^{2}\right\|_{H^{1}}\right)\right\} .
\end{aligned}
$$

This expression approaches 0 as $n \rightarrow \infty$, because $w_{n}$ and $w \in H^{1}(\mathbb{R})$ imply $\left\|w^{r} w_{n}^{s}\right\|_{H^{1}}<\infty$ for $r, s=0,1,2$; Thus, $\lim _{n \rightarrow \infty}\left|E\left(w_{n}\right)-E(w)\right|=0$.

It is well known that the initial value problem for (1.1) is globally well posed. In fact, as soon as local existence is established, the conservation of the $H^{1}$-norm can be exploited to obtain a global solution. For the exact proof, the reader may consult the articles of Benjamin et al. [6] and Albert and Bona [3].

The notation used in this article is the standard notation in the theory of partial differential equations. Since all functions considered here are real-valued, we take the $L^{2}$-inner product to be $\langle f, g\rangle=\int_{-\infty}^{\infty} f(x) g(x) d x$. We will also have occasion to consider the $L^{2}$-inner product on the half-line, and this will be denoted by $\langle f, g\rangle_{L^{2}[0, \infty)}=\int_{0}^{\infty} f(x) g(x) d x$.

\section{Orbital stability}

In this section, orbital stability of the solitary waves of depression will be proved. Consider for a moment the difference in $L^{2}(\mathbb{R})$ of a solitary wave and a general solution of (1.1). Intuitively, for each $u$ in $H^{1}(\mathbb{R})$, there is an $\alpha \in \mathbb{R}$, such that

$$
\int_{-\infty}^{\infty}\{u(\xi+\alpha(u))-\Phi(\xi)\}^{2} d \xi=\inf _{a \in \mathbb{R}} \int_{-\infty}^{\infty}\{u(\xi+a)-\Phi(\xi)\}^{2} d \xi .
$$


If the integral on the right is a differentiable function of $a$, and $\|u\|_{L^{2}}=\|\Phi\|_{L^{2}}$, then $\alpha(u)$ could be determined by solving the equation

$$
\left\langle u(\cdot+\alpha(u)), \Phi^{\prime}\right\rangle=0 .
$$

A formal proof of the existence of a $\alpha(u)$ proceeds with the use the implicit function theorem as follows.

Proposition 3.1. There is $\epsilon>0$, such that there exists a $C^{1}$-mapping $\alpha: U_{\epsilon} \longrightarrow \mathbb{R}$, with the property that $\left\langle u(\cdot+\alpha(u)), \Phi^{\prime}\right\rangle=0$ for every $u \in U_{\epsilon}$.

Proof. For a given $u \in U_{\epsilon}$, consider the functional

$$
F:(u, \alpha) \longmapsto \int_{-\infty}^{\infty} u(\xi+\alpha(u)) \Phi^{\prime}(\xi) d \xi
$$

Observe that

$$
\frac{d F(\Phi, 0)}{d \alpha}=\int_{-\infty}^{\infty}\left(\Phi^{\prime}(\xi)\right)^{2} d \xi>0
$$

and

$$
F(\Phi, 0)=\int_{-\infty}^{\infty} \Phi(\xi) \Phi^{\prime}(\xi) d \xi .=\frac{1}{2} \int_{-\infty}^{\infty} \frac{d}{d \xi} \Phi^{2}(\xi) d \xi=\frac{1}{2}\left[\Phi^{2}(\infty)-\Phi^{2}(-\infty)\right]=0
$$

Therefore, by the implicit function theorem, there exist a $C^{1}$-map $\alpha(u)$ near $\Phi$ such that

$$
\left\langle u(\cdot+\alpha(u)), \Phi^{\prime}\right\rangle=0 .
$$

By translation invariance, the size of the neighborhood is the same everywhere.

A crucial ingredient in the proof of stability is the fact that for all $c$ less than some critical speed $c^{*}$, the functional $E(u)$ attains its minimum value when restricted to functions for which $V(u)=V(\Phi)$. In fact, we have the following explicit estimate.

Proposition 3.2. If $c<-\frac{1}{6}$, there are $\beta>0$, and $\epsilon>0$ such that

$$
E(u)-E(\Phi) \geq \frac{\beta}{2}\|u(\cdot+\alpha(u))-\Phi\|_{H^{1}}^{2},
$$

for all $u \in U_{\epsilon}$, satisfying $V(u)=V(\Phi)$.

Proof. The demonstration of this theorem follows the ideas outlined in the work of Benjamin [5]. In that work, however, the focus was on positive solitary waves. To accommodate negative solitary waves, the proof has to be modified accordingly.

For each $u$ in $U_{\epsilon}$ such that $V(u)=V(\Phi)$, let $v=u(\cdot+\alpha(u))-\Phi$, where $\alpha$ is defined in Proposition 3.1. Let $\Delta V=V(\Phi+v)-V(\Phi)$, and note that $\Delta V=0$. However, according to the definition of $V$, we also have

$$
\Delta V=\frac{1}{2} \int_{-\infty}^{\infty}\left\{v^{2}+v^{\prime 2}+2 \Phi v+2 \Phi^{\prime} v^{\prime}\right\} d \xi
$$


Defining $\Delta E$ in a similar way, we see that

$$
\Delta E=E(\Phi+v)-E(\Phi)=\int_{-\infty}^{\infty}\left\{\frac{1}{2} v^{2}+\Phi v+\Phi^{2} v+\Phi v^{2}+\frac{1}{3} v^{3}\right\} d \xi
$$

On the other hand, since $\Delta V=0$, we may also write

$$
\Delta E=\Delta E-c \Delta V \text {. }
$$

Therefore, in view of equations (3.3) and (3.4) there appears the expression

$$
\begin{aligned}
\Delta E= & \int_{-\infty}^{\infty}\left\{\frac{1}{2} v^{2}+\Phi v^{2}+\frac{1}{3} v^{3}-\frac{1}{2} c v^{2}-\frac{1}{2} c v^{\prime 2}\right\} d \xi \\
& +\int_{-\infty}^{\infty}\left\{\Phi-c \Phi+\Phi^{2}+c \Phi^{\prime \prime}\right\} v d \xi .
\end{aligned}
$$

Since $\Phi$ satisfies (1.2), the integrand of the second integral vanishes identically. Therefore,

$$
\Delta E=\frac{1}{2} \int_{-\infty}^{\infty}\left\{-c v^{\prime 2}+(-c+1+2 \Phi) v^{2}\right\} d \xi+\frac{1}{3} \int_{-\infty}^{\infty} v^{3} d \xi
$$

Thus we have $\Delta E=\delta^{2} E+\delta^{3} E$, where $\delta^{2} E$ and $\delta^{3} E$ are the second and third variation of $E$, respectively. In order to obtain a lower bound for $\delta^{2} E, v$ is written as the sum of an even function $f$ and an odd function $g$. Since $\Phi$ itself is even, it can be shown directly that the even and odd parts of $v$ contribute independently to $\delta^{2} E$. In other words, we have

$$
\delta^{2} E=\delta^{2} E(f)+\delta^{2} E(g) .
$$

The contribution to $\delta^{2} E$ from even functions is obtained as follows.

Lemma 3.3. If $c<-\frac{1}{6}$, there are positive constants $\kappa_{1}$ and $\kappa_{2}$ such that

$$
\delta^{2} E(f) \geq \kappa_{1}\|f\|_{H^{1}}^{2}-\kappa_{2}\|v\|_{H^{1}}^{3} .
$$

Proof. The estimate for the lower bound of the contribution of $\delta^{2} E(f)$, where $f$ is an even function will be obtained by comparison with the integral

$$
J=\int_{0}^{\infty}\left\{2 \sqrt{\frac{c}{c-1}} f^{\prime 2}+\left[\frac{\mu}{2} \sqrt{\frac{c-1}{c}}+\frac{20}{3} \sqrt{\frac{1}{c(c-1)}} \Phi\right] f^{2}\right\} d \xi
$$

where $\mu$ is a constant to be specified later. The substitution $z=\frac{1}{2} \sqrt{\frac{c-1}{c}} \xi$ yields $\Phi=\frac{3}{2}(c-1) \operatorname{sech}^{2} z$, and puts the integral in the simpler form

$$
J=\int_{0}^{\infty}\left\{\left(\frac{\partial f}{\partial z}\right)^{2}+\left(\mu-20 \operatorname{sech}^{2} z\right) f^{2}\right\} d z
$$

From here one, derivatives with respect to $z$ will be denoted by $\frac{\partial}{\partial z}$, while derivatives with respect to $\xi$ will be indicated by a prime. Moreover, the integrands will be interpreted as functions of $z$ or $\xi$ as indicated by the variable of integration. Next, 
we will make use of the spectral theory for a certain linear operator on the Hilbert space $L^{2}(0, \infty)$. Let the operator $\mathcal{L}_{e}$ be defined by

$$
\mathcal{L}_{e}=-\frac{d^{2}}{d z^{2}}-20 \operatorname{sech}^{2} z
$$

with domain defined as those functions $\phi \in H^{2}(0, \infty)$ that satisfy the boundary condition $\phi^{\prime}(0)=0$. As it turns out, $\mathcal{L}_{e}$ has only two negative eigenvalues:

$$
\lambda_{1}=-16 \text { and } \lambda_{2}=-4,
$$

with corresponding normalized eigenfunctions

$$
\begin{aligned}
& \psi_{1}=\sqrt{\frac{35}{16}} \operatorname{sech}^{4} z \text {, and } \psi_{2}=\sqrt{\frac{5}{8}}\left(6 \operatorname{sech}^{2} z-7 \operatorname{sech}^{4} z\right) . \\
& \underset{\lambda_{1}=-16}{\lambda_{2}=-4} \underset{0}{\bullet}-\underbrace{}_{0} \lambda>0
\end{aligned}
$$

Figure 2. The spectrum of $\mathcal{L}_{e}$.

The rest of the singular set consists of positive continuous spectrum. Now it can be seen from the expressions in (3.7) that $\psi_{1}$ and $\psi_{2}$ have $\operatorname{sech}^{4} z$ as a common term. Thus we may write $\operatorname{sech}^{2} z$ as a linear combination of $\psi_{1}$ and $\psi_{2}$. In particular,

$$
\operatorname{sech}^{2} z=\frac{7}{6} \sqrt{\frac{16}{35}} \psi_{1}+\frac{1}{6} \sqrt{\frac{8}{5}} \psi_{2} .
$$

The connection between $J$ and $\mathcal{L}_{e}$ becomes apparent as follows.

$$
\left\langle\mathcal{L}_{e} f, f\right\rangle_{L^{2}[0, \infty)}=\int_{0}^{\infty}\left\{f^{\prime 2}-20 \operatorname{sech}^{2} z f^{2}\right\} d z
$$

One should recognize the right-hand side of this equation as the first and last term in the integral $J$. By the spectral theorem, the left-hand side of this equation is equal to

$$
-16 F_{1}^{2}-4 F_{2}^{2}+\int_{0}^{\infty} F^{2}(\lambda) \lambda d \rho(\lambda),
$$

where $\rho(\lambda)$ is the spectral-function on $\mathbb{R}$. The coefficients $F_{1}, F_{2}$, and $F(\lambda)$ are defined by

$$
F_{1,2}=\int_{0}^{\infty} \psi_{1,2} f(z) d z \text { and } F(\lambda)=\int_{0}^{\infty} \psi(z ; \lambda) f(z) d z
$$

where $\psi(z ; \lambda)$ is the generalized eigenfunction corresponding to the continuous spectrum of $\mathcal{L}_{e}$. Moreover, the spectral decomposition

$$
f(z)=F_{1} \psi_{1}+F_{2} \psi_{2}+\int_{0}^{\infty} \psi(z ; \lambda) F(\lambda) d \rho(\lambda),
$$


and Parseval's identity give

$$
\int_{0}^{\infty} f^{2} d z=F_{1}^{2}+F_{2}^{2}+\int_{0}^{\infty} F^{2} d \rho(\lambda) .
$$

From equation (3.9) and (3.11), we obtain a new form for the integral $J$ as follows:

$$
J=(\mu-16) F_{1}^{2}+(\mu-4) F_{2}^{2}+\int_{0}^{\infty}(\mu+\lambda) F^{2} d \rho(\lambda) .
$$

Next, we introduce the notation $p=\|v\|_{H^{1}}$, and write $F_{2}$ as a linear combination of $F_{1}$ and $p^{2}$. Here, the constraint $\Delta V=0$ will play a major role. According to equation (3.3), the constraint $\Delta V=0$ is equivalent to

$$
-p^{2}=2 \int_{-\infty}^{\infty}\left(\Phi v+\Phi^{\prime} v^{\prime}\right) d \xi
$$

On the right-hand side, use integration by parts in the last term yields

$$
-p^{2}=2 \int_{-\infty}^{\infty}\left(\Phi-\Phi^{\prime \prime}\right) v d \xi .
$$

In light of equation (1.2) and the fact that $\Phi$ is an even function, this equation can be put in the form

$$
-p^{2}=\frac{4}{c} \int_{0}^{\infty}\left(\Phi+\Phi^{2}\right) f d \xi .
$$

On the right-hand side of this equation, use the expression (1.3) for $\Phi$, and make a change variable $z=\frac{1}{2} \sqrt{\frac{c-1}{c}} \xi$. Then (3.7) and (3.10) can be used to put the equation in the form of

$$
\int_{0}^{\infty} \operatorname{sech}^{2} z f d z=-\frac{3}{2} \sqrt{\frac{16}{35}}(c-1) F_{1}-\frac{1}{12} \sqrt{\frac{c}{c-1}} p^{2} .
$$

Using the definition of $F_{2},(3.13)$, the definition of $F_{1}$, it appears that

$$
F_{2}=A F_{1}+I p^{2}
$$

where $A$ and $I$ are defined by

$$
A=(-9 c+2) \sqrt{\frac{2}{7}}, \text { and } I=-\frac{1}{2} \sqrt{\frac{5}{8}} \sqrt{\frac{c}{c-1}} .
$$

Thus equation (3.12) becomes

$$
J=\left[\mu-16+(\mu-4) A^{2}\right] F_{1}^{2}+(\mu-4)\left(2 A I F_{1} p^{2}+I^{2} p^{4}\right)+\int_{0}^{\infty}(\mu+\lambda) F^{2} d \rho(\lambda) .
$$

Now for positive $\mu$, the integral term is nonnegative. We choose $\mu$ is such a way that the coefficient of $F_{1}^{2}$ in the expression for $J$ is nonnegative. Thus we need

$$
\mu \geq \frac{4\left(4+A^{2}\right)}{1+A^{2}} .
$$


Since $\frac{4\left(4+A^{2}\right)}{1+A^{2}}>4$, the coefficient $(\mu-4)$ is then automatically also positive. Therefore, with this choice of $\mu, J$ can be estimated below by

$$
J \geq \frac{24}{1+A^{2}} A I F_{1} p^{2} .
$$

By using straightforward inequalities

$$
\begin{aligned}
F_{1}^{2} \leq \int_{0}^{\infty} f^{2} d z \leq \frac{1}{2} \int_{-\infty}^{\infty} v^{2} d z & \leq \frac{1}{2} \int_{-\infty}^{\infty}\left\{v^{2}+\frac{1}{4} \frac{c-1}{c} v^{\prime 2}\right\} d z \\
& =\frac{1}{4} \sqrt{\frac{c-1}{c}} \int_{-\infty}^{\infty}\left\{v^{2}+v^{\prime 2}\right\} d \xi=\frac{1}{4} \sqrt{\frac{c-1}{c}} p^{2}
\end{aligned}
$$

we obtain the lower bound for the integral as

$$
J \geq \frac{12 A I}{1+A^{2}}\left(\frac{c-1}{c}\right)^{\frac{1}{4}}\|v\|_{H^{1}}^{3} .
$$

Finally, a lower bound for the even contribution to $\delta^{2} E$ is found as follows.

$$
\begin{aligned}
\delta^{2} E(f)= & \int_{0}^{\infty}\left\{-\frac{2 c}{5} f^{\prime 2}+(-c+1)\left(1-\frac{3}{20} \mu\right) f^{2}\right\} d \xi+\frac{3}{10} \sqrt{c(c-1)} J \\
\geq & \frac{1}{2} \int_{-\infty}^{\infty}\left\{-\frac{2 c}{5} f^{\prime 2}+(-c+1)\left(1-\frac{3}{20} \mu\right) f^{2}\right\} d \xi \\
& +\frac{18 A I}{5\left(1+A^{2}\right)} \sqrt{c(c-1)}\left(\frac{c-1}{c}\right)^{\frac{1}{4}}\|v\|_{H^{1}}^{3} .
\end{aligned}
$$

Now we need the coefficient $1-\frac{3}{20} \mu$ to be positive, and considering (3.15), this is possible only if $\frac{20}{3}>\mu \geq \frac{4\left(4+A^{2}\right)}{1+A^{2}}$. But by (3.14), this inequality can be satisfied only if $c<-\frac{1}{6}$. Thus as long as $c<-\frac{1}{6}$, we have the estimate

$$
\delta^{2} E(f) \geq \kappa_{1} \int_{-\infty}^{\infty}\left(f^{\prime 2}+f^{2}\right) d \xi-\kappa_{2}\|v\|_{H^{1}}^{3}
$$

where $\kappa_{1}=\min \left(-\frac{c}{5}, \frac{1}{2}(-c+1)\left(1-\frac{3}{20} \mu\right)\right)$, and $\kappa_{2}=\frac{-18 A I}{5\left(1+A^{2}\right)} \sqrt{c(c-1)}\left(\frac{c-1}{c}\right)^{\frac{1}{4}}$ are positive constants.

Next, we turn to the contribution to $\delta^{2} E$ from the odd part of $v$.

Lemma 3.4. For all $c<0$, there holds the estimate

$$
\delta^{2} E(g) \geq \frac{-c}{8}\|g\|_{H^{1}}^{2} .
$$

Proof. For the odd contribution to $\delta^{2} E$, the result in Proposition 3.1 will play an important role. By virtue of this lemma

$$
\int_{-\infty}^{\infty}(\Phi+v) \Phi^{\prime} d \xi=0 .
$$


Since $\Phi$ is even, this is the same as

$$
\int_{-\infty}^{\infty} g \Phi^{\prime} d \xi=0
$$

Let $s$ be a positive constant, and consider the linear operator

$$
\mathscr{L}_{o}=\frac{1}{s}\left[-\frac{d^{2}}{d \xi^{2}}-3 s \operatorname{sech}^{2}\left(\frac{1}{2} \sqrt{s} \xi\right)\right],
$$

defined on those functions $\phi \in H^{2}(0, \infty)$ that satisfy the boundary condition $\phi(0)=0$. As illustrated in Figure $3, \mathscr{L}_{o}$ has only one negative eigenvalue $\lambda_{1}=-1$ with corresponding eigenfunction

$$
\theta_{1}=\frac{d}{d \xi}\left\{3 s \operatorname{sech}^{2}\left(\frac{1}{2} \sqrt{s} \xi\right)\right\},
$$

and the rest of the spectrum of $\mathscr{L}_{o}$ is positive continuous [5].

$$
\lambda_{1}=-1 \quad \text { Positive continuous spectrum } \lambda>0
$$

Figure 3. The spectrum of $\mathscr{L}_{0}$.

Now the spectral theorem asserts that

$$
\left\langle\mathscr{L}_{o} g, g\right\rangle_{L^{2}[0, \infty)}=-\left\langle g, \theta_{1}\right\rangle_{L^{2}[0, \infty)}^{2}+\int_{0}^{\infty} \lambda G^{2}(\lambda) d \rho(\lambda),
$$

where $G(\lambda)=\int_{0}^{\infty} \theta(\xi ; \lambda) g(\xi) d \xi$, and $\theta(\xi ; \lambda)$ is a generalized eigenfunction of $\mathscr{L}_{o}$. If we now choose $s=\frac{c-1}{c}$, where $c<0$ then it follows from (3.18) that

$$
\left\langle g, \theta_{1}\right\rangle_{L^{2}[0, \infty)}=0 \text {. }
$$

Thus we obtain

$$
\left\langle\mathscr{L}_{o} g, g\right\rangle_{L^{2}[0, \infty)} \geq 0
$$

On the other hand, after integration by parts, there appears

$$
0 \leq\left\langle\mathscr{L}_{o} g, g\right\rangle_{L^{2}[0, \infty)}=\frac{c}{c-1} \int_{0}^{\infty}\left\{g^{2}-3 \frac{c-1}{c} \operatorname{sech}^{2}\left(\frac{1}{2} \sqrt{\frac{c-1}{c}} \xi\right) g^{2}\right\} d \xi .
$$

Thus it is immediate that the following integral is nonnegative.

$$
K=\int_{0}^{\infty}\left\{g^{\prime 2}-\frac{2}{c} \Phi g^{2}\right\} d \xi \geq 0 .
$$

The contribution to $\delta^{2} E$ due to the odd part of $v$ may now be estimated by comparison with the integral $K$.

$\delta^{2} M(g)=-\frac{3 c}{4} K+\frac{1}{2} \int_{0}^{\infty}\left\{\Phi+\frac{3}{2}(-c+1)\right\} g^{2} d \xi+\frac{1}{4} \int_{0}^{\infty}\left\{-c g^{\prime 2}+(-c+1) g^{2}\right\} d \xi$. 
In light of (3.22), $-c+1 \geq-c$, and the fact that $\Phi \geq \frac{3}{2}(c-1)$ [cf.(1.3) with $c<0$ ], there follows

$$
\delta^{2} M(g) \geq \frac{-c}{8} \int_{-\infty}^{\infty}\left(g^{\prime 2}+g^{2}\right) d \xi .
$$

Proof of Proposition 3.2: Using (3.5), Lemma (3.3), and Lemma (3.4), it is plain that

$$
\delta^{2} E \geq \beta \int_{-\infty}^{\infty}\left(v^{\prime 2}+v^{2}\right) d \xi-\kappa_{2}\|v\|_{H^{1}}^{3},
$$

where $\beta=\min \left(\frac{-c}{8}, \kappa_{1}\right)$ is a positive constant, and $\kappa_{1}$ and $\kappa_{2}$ are defined in (3.17).

Now using the inequality $\sup _{\xi \in \mathbb{R}}|v(\xi)| \leq \frac{1}{\sqrt{2}}\|v\|_{H^{1}}{ }^{1}$, there appears an estimate for $\delta^{3} E$.

$$
\delta^{3} E=-\frac{1}{3} \int_{-\infty}^{\infty}(-v) v^{2} d \xi \geq-\frac{1}{3} \sup |v| \int_{-\infty}^{\infty} v^{2} d \xi \geq-\frac{1}{3 \sqrt{2}}\|v\|_{H^{1}}^{3} .
$$

Combining (3.24) and (3.25) yields the final estimate

$$
\Delta E \geq \beta \int_{-\infty}^{\infty}\left(v^{\prime 2}+v^{2}\right) d \xi-\gamma\|v\|_{H^{1}}^{3}=\|v\|_{H^{1}}^{2}\left(\beta-\gamma\|v\|_{H^{1}}\right) .
$$

where $\gamma=\kappa_{2}+\frac{1}{3 \sqrt{2}}$. Therefore, if $\|v\|_{H^{1}}$ is sufficiently small, say $\|v\|_{H^{1}}<\frac{\beta}{2 \gamma}$, we obtain

$$
\Delta E \geq \frac{\beta}{2}\|v\|_{H^{1}}^{2}
$$

Finally, we will close this section by showing a necessary condition for stability of the solitary-wave.

Theorem. The solitary wave $\Phi$ with velocity $c$ is stable if $c<-\frac{1}{6}$.

Proof. The proof is based on the techniques of of Bona, Grillakis, Souganidis Shatah, and Strauss in $[8,10]$. In particular, the theorem will be proved by contradiction as follows. Suppose $\Phi$ is not stable, then there exists an $\epsilon>0$, and a sequence of initial data $u_{n}^{0} \in H^{1}(\mathbb{R})$ and corresponding solutions $u_{n} \in C\left(\mathbb{R}, H^{1}(\mathbb{R})\right)$ with $u_{n}(\cdot, 0)=u_{0}^{n}$, such that

$$
\lim _{n \rightarrow \infty}\left\|u_{n}^{0}-\Phi\right\|_{H^{1}}=0
$$

but

$$
\sup _{t>0} \inf _{s \in \mathbb{R}}\left\|u_{n}(\cdot, t)-\tau_{s} \Phi(\cdot)\right\|_{H^{1}} \geq \frac{1}{2} \epsilon,
$$

for large enough $n$. By the continuity of $u_{n}$ in t, we can pick the first time $t_{n}$ so that

$$
\inf _{s \in \mathbb{R}}\left\|u_{n}\left(\cdot, t_{n}\right)-\tau_{s} \Phi(\cdot)\right\|_{H^{1}}=\frac{1}{2} \epsilon .
$$

In other words, $u_{n}\left(\cdot, t_{n}\right) \in \partial U_{\frac{1}{2} \epsilon}$.

\footnotetext{
${ }^{1}$ This is known as the Sobolev lemma. The reader may refer to [5] for a simple proof.
} 
Since $V$ is continuous in $H^{1}(\mathbb{R})$ and invariant under time evolution, we have $\lim _{n \rightarrow \infty} V\left(u_{n}^{0}\right)=V(\Phi)$, and consequently

$$
\lim _{n \rightarrow \infty} V\left(u\left(\cdot, t_{n}\right)\right)=V(\Phi) .
$$

Choose a sequence $w_{n} \in H^{1}(\mathbb{R})$, such that $V\left(w_{n}\right)=V(\Phi)$ and $\lim _{n \rightarrow \infty} \| w_{n}-$ $u_{n}\left(\cdot, t_{n}\right) \|_{H^{1}}=0 .{ }^{2}$ Note that by $H^{1}$-continuity of $E$, and time invariance,

$$
\lim _{n \rightarrow \infty}\left[E\left(w_{n}\right)-E(\Phi)\right]=0,
$$

and also note that $w_{n} \in U_{\epsilon}$ for large $n$. On the other hand, so long as $\epsilon$ is small enough, Proposition (3.2) shows that

$$
E\left(w_{n}\right)-E(\Phi) \geq \frac{\beta}{2}\left\|w_{n}\left(\cdot+\alpha\left(w_{n}\right)\right)-\Phi\right\|_{H^{1}}^{2},
$$

where $\beta$ is the constant defined in (3.24). Therefore, since $\alpha(u)$ is a continuous function, it appears that

$$
\lim _{n \rightarrow \infty}\left\|u_{n}\left(\cdot, t_{n}\right)-\Phi\left(\cdot-\alpha\left(u_{n}\left(\cdot, t_{n}\right)\right)\right)\right\|_{H^{1}}=0 .
$$

Finally, this is a contradiction to (3.27)

\section{References}

[1] J.P. Albert, Concentration compactness and the stability of solitary-wave solutions to nonlocal equations. Applied analysis (Baton Rouge, LA, 1996), 1-29, Contemp. Math. 221, Amer. Math. Soc., Providence, RI, 1999.

[2] J.P. Albert and J.L. Bona, Total positivity and the stability of internal waves in stratified fluids of finite depth. The Brooke Benjamin special issue (University Park, PA, 1989). IMA J. Appl. Math. 46 (1991), 1-19.

[3] J.P. Albert and J.L. Bona, Comparisons between model equations for long waves. J. Nonlinear Sci. 1 (1991), 345-374.

[4] J.P. Albert and J.L. Bona and D.B. Henry, Sufficient conditions for stability of solitary-wave solutions of model equations for long waves. Phys. D 24 (1987), 343366 .

[5] T.B. Benjamin, The stability of solitary waves. Proc. Roy. Soc. London A 328 (1972), 153-183.

[6] T.B. Benjamin, J.B. Bona, and J.J. Mahony, Model equations for long waves in nonlinear dispersive systems. Philos. Trans. Roy. Soc. London A 272 (1972), 47-78.

[7] J.L. Bona, On the stability theory of solitary waves. Proc. Roy. Soc. London A 344 (1975), 363-374.

[8] J.L. Bona, P.E. Souganidis and W.A. Strauss, Stability and instability of solitary waves of Korteweg-de Vries type. Proc. Roy. Soc. London A 411 (1987), 395-412.

\footnotetext{
${ }^{2}$ The sequence defined by $w_{n}=\left(\|\Phi\|_{H^{1}} /\left\|u_{n}\right\|_{H^{1}}\right) u_{n}$ will do the job.
} 
[9] J. Boussinesq, Théorie des ondes et des remous qui se propagent le long d'un canal rectangulaire horizontal, en communiquant au liquide contenu dans ce canal des vitesses sensiblement pareilles de la surface au fond. J. Math. Pures Appl. 17 (1872), 55-108.

[10] M. Grillakis, J. Shatah and W.A. Strauss, Stability theory of solitary waves in the presence of symmetry. J. Funct. Anal. 74 (1987), 160-197.

[11] I.D. Iliev, E.Kh. Khristov and K.P. Kirchev, Spectral methods in soliton equations. Pitman Monographs and Surveys in Pure and Applied Mathematics 73. Longman Scientific \& Technical, Harlow; copublished in the United States with John Wiley \& Sons, Inc., New York, 1994.

[12] H. Kalisch, Solitary Waves of Depression. J. Computational Analysis and Application 8 (2006), 5-24.

[13] N. Nguyen and H. Kalisch, Orbital Stability of Negative Solitary Waves, submitted.

[14] P.G. Peregrine, Calculations of the development of an undular bore. J. Fluid Mech. 25 (1966), 321-330.

[15] J. Shatah and W. Strauss, Instability of nonlinear bound states. Commun. Math. Phys. 100 (1985), 173-190.

[16] P.E. Souganidis and W.A. Strauss, Instability of a class of dispersive solitary waves. Proc. Roy. Soc. Edinburgh 114A (1990), 195-212.

[17] G.B. Whitham, Linear and Nonlinear Waves. Wiley, New York, 1974.

Nguyen Thanh Nguyet

Department of Mathematics

University of Bergen

Johannes Brunsgate 12

5008 Bergen, Norway

e-mail: Nguyet.Nguyen@math.uib.no

Henrik Kalisch

Department of Mathematics

University of Bergen

Johannes Brunsgate 12

5008 Bergen, Norway

e-mail: henrik.kalisch@math.uib.no 